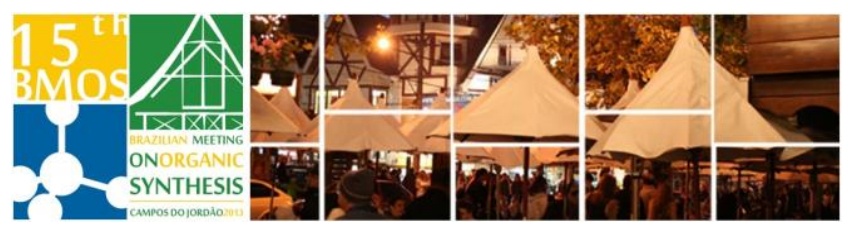

\title{
Study of the ortho-methoxy substituent group effect in selective demethylation reactions of methoxybenzoic acids.
}

\author{
Rafael D. C. Gallo, Diandra P. Alencar, Murilo Yonekawa and Rozanna M. Muzzi
}

Faculdade de Ciências Exatas e Tecnologia, Universidade Federal da Grande Dourados, Rodovia Dourados-Itahum, Km 12, Cidade Universitária, Dourados, MS, CEP 79.804-970, Brazil

*e-mail address: rozannamuzzi@ufgd.edu.br

Keywords: demethylation, decarboxylation and ortho effect

\section{INTRODUCTION}

The methylation of the hydroxyl group is largely used in synthesis due to high stability of the methoxy group to several reaction conditions. However, the procedures for $\mathrm{O}$-demethylation of aryl methyl ethers involves the use of costly reagents or elaborated methods, requiring anhydrous conditions or difficult to carry out. In most cases the simplest methods of $O$ demethylation are not selective, resulting in complete demethylation products $^{1,2}$. In order to prepare demethylated 3,5-dimethoxybenzoic derivatives, this work presents a new selective methodology for demethylation of phenolic methoxyl group using simple and inexpensive reagents. The methodology was adjusted to 3,5-dimethoxybenzoic acid and is summarized in the reaction of aryl methyl ether with ethylene glycol, $\mathrm{KOH}$, at $120^{\circ} \mathrm{C}$ or $180^{\circ} \mathrm{C}$.

\section{RESULTS AND DISCUSSION}

Initial testing were carried out with 3,5dimethoxybenzoic acid, we have determined that the entry 7 has been the best condition found for the highest yield of the desired product (Table 1). This methodology was also applied to 2,3-, 2,4- and 2,6dimethoxybenzoic acids; 4-methoxybenzoic and 4-bromo-3,5-dimethoxybenzoic acids. In such cases it was possible to observe that the reaction has proceeded best at $180{ }^{\circ} \mathrm{C}$ and in the ortho or paramethoxyl substrates was observed the formation of decarboxylation products (Table 2).

Table 1. Monodemethylation reaction conditions for 3,5dimethoxybenzoic acid using ethylene glycol or diethylene glycol and $\mathrm{KOH}$.

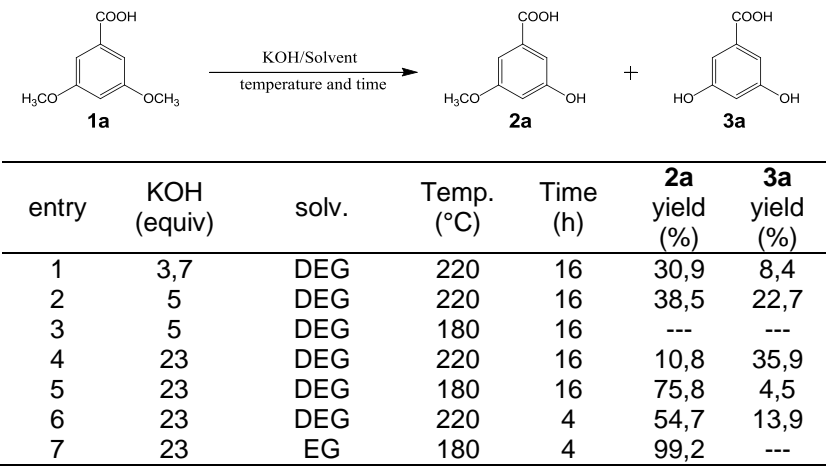

$\mathrm{DEG}=$ Diethylene glycol $\quad \mathrm{EG}=$ Ethylene glycol
Table 2. O-demethylation of aryl methyl ethers using ethylene glycol and $\mathrm{KOH}$.

\begin{tabular}{|c|c|c|c|c|c|c|}
\hline entry & diethers & $\begin{array}{c}\mathrm{KOH} \\
\text { (equiv) }\end{array}$ & $\begin{array}{c}\text { Temp } \\
\left({ }^{\circ} \mathrm{C}\right)\end{array}$ & $\begin{array}{c}\text { Time } \\
\text { (h) }\end{array}$ & Product & yield $(\%)$ \\
\hline 1 & 1b & 23 & 180 & 4 & $2 b$ & 100 \\
\hline 2 & 1c & 23 & 180 & 4 & $2 c$ & 99 \\
\hline 3 & 1d & 23 & 180 & 4 & $2 d$ & 92 \\
\hline 4 & 1e & 23 & 180 & 4 & $2 e$ & 99 \\
\hline 5 & 1f & 23 & 180 & 4 & $2 f$ & 96 \\
\hline 6 & $1 \mathrm{~g}$ & 10 & 180 & 4 & $\begin{array}{l}\mathrm{Br} \\
2 \mathrm{~g}\end{array}$ & 89 \\
\hline
\end{tabular}

\section{CONCLUSION}

This methodology resulted in the selective demethylation of aryl methyl ethers and the desired products were obtained in good yields. However, in substrates with a methoxy group at the ortho position products of decarboxylation were produced too.

\section{ACKNOWLEDGEMENTS}

We acknowledge CNPq and FUNDECT for financial support.

\section{REFERENCES}

${ }^{1}$ Kim Y. I.; Kim Y. H. Tetrahedron Lett. 1998, 39, 639.

${ }^{2}$ Fang Z.; Zhou G.; Zheng Z.; He G.; Li J.; He L.; Bei D. Journal of Molecular Catalysis A: Chemical, 2007, 274,16. 